\title{
Synchronous intercept strategies for a robotic defense-intrusion game with two defenders
}

\author{
Shuai Zhang ${ }^{1} \cdot$ Mingyong Liu $^{1} \cdot$ Xiaokang Lei ${ }^{2,3}$ - Panpan Yang ${ }^{4}$. \\ Yunke Huang $^{1}$ • Ruaridh Clark ${ }^{5}$
}

Received: date / Accepted: date

\begin{abstract}
We study the defense-intrusion game, in which a single attacker robot tries to reach a stationary target that is protected by two defender robots. We focus on the "synchronous intercept problem", where both robots have to reach the attacker robot synchronous$l y$ to intercept it. Assume that the attacker robot has the control policy which is based on attraction to the target and repulsion from the defenders, two kinds of synchronous intercept strategies are proposed for the defense-intrusion game, introduced here as Attackeroriented and Neutral-position-oriented. Theoretical analysis and simulation results show that: (1) The two strategies are able to generate different synchronous intercept patterns: contact intercept pattern and stable non-contact intercept pattern, respectively. (2) The contact intercept pattern allows the defender robots to intercept the attacker robot in finite time, while the stable non-contact intercept pattern generates a periodic attractor that prevents the attack robot from reaching the target for infinite time. There is potential to apply the insights obtained into defense-intrusion in real system$\mathrm{s}$, including aircraft escort and the defense of military targets or territorial boundaries.
\end{abstract}

Shuai Zhang

E-mail: shuaizhang@mail.nwpu.edu.cn

1 School of Marine Science and Technology, Northwestern Polytechnical University, Xi'an, Shaanxi, P. R. China, 710072

${ }^{2}$ School of Information and Control Engineering, Xi'an University of Architecture and Technology, Xi'an, Shaanxi, P. R. China, 710055

${ }^{3}$ KLINNS Lab, Xi'an Jiaotong University, Xi'an, Shaanxi, P. R. China

${ }^{4}$ School of Electronic and Control Engineering, Chang'an University, Xi'an, China

${ }^{5}$ Department of Mechanical and Aerospace Engineering, University of Strathclyde, Glasgow, UK
Keywords Pursuit-evasion · Defense-intrusion . Synchronous intercept $\cdot$ Multi-robot systems

\section{Introduction}

Pursuit-evasion phenomena is widely observed in nature, an example of which is the interaction between coyotes, elk and wolves in Yellowstone National Park (Ripple and Larsen, 2000; Gese, 2001). Studying this phenomena provides an insight into natural interactions, such as prey escape strategies (Breakwell, 1975; Bhattacharya et al, 2011, 2014; Yang et al, 2014; Zha et al, 2016; Zhang et al, 2019), collective behavior (Neill and Cullen, 1974; Siegfried and Underhill, 1975; Bertram, 1978), catching efficiency for predators (Iwama and Sato, 2012; Saito et al, 2016; Masuko et al, 2017; Janosov et al, 2017) and the optimal number of predators for predation success (Kamimura and Ohira, 2010; Vicsek, 2010). But this approach can also provide elegant solutions for artificial systems, including the design of target trapping by autonomous robots (Antonelli et al, 2007; Huang et al, 2013; Peng et al, 2016; Zhang et al, 2018).

A distinct but similar problem is that of defenseintrusion, another interesting interaction that is widely observed in the animal world (e.g., a lioness tries to protect her cubs against a predator or predators.) and in artificial systems (e.g., a group of guard robots try to protect the base against the intruders). It is important to note that this class of games are different from pursuit-evasion games. The main difference is that one set of players must not only consider the other set of players, but also the target (Zhou et al, 2018). The Target-Attacker-Defender (TAD) game (Boyell, 1976) is a game that simulates this defense-intrusion inter- 
action, where there are three different robots - Target, Attacker, and Defender. In this game the attacker robot aims to capture the target robot, while avoiding being caught by the defender robot. The defender robot tries to defend the target robot, while avoiding being destroyed by the attacker robot but is also tasked with trying to capture the attacker robot at an opportune moment.

We extend the TAD game with respect to synchronous intercept. Two homogeneous autonomous robots are regarded as the defenders, they aim to reach and capture the attacker robot synchronously, if either of the two defender robots reach (attack) the attacker robot independently then it is destroyed. The motivation for this extension is the scenario where a single defender robot is insufficient to capture, or compel the attacker robot to abandon its invasion. In this situation, the defender robots need a collective intercept strategy to achieve their objectives. This scenario is seen in nature when the prey is often weaker than the predator but can overcome their attacker when acting collectively. A similar scenario can be envisioned for guard drones that can only stop a well defended attacking vehicle if they intercept synchronously. Our motivation is similar to the work in Bopardikar and Suri (2014), where a special pursuit-evasion game is referred to as $k$-capture. In this $k$-capture game, the pursuers and the evader move with the same maximum speed, and at least $k$ pursuers must simultaneously reach the evader's location to capture it.

Our objective in this paper is to study the intercept strategies for the defender robots, as they attempt a synchronous intercept mission based on the assumption that the attacker robot has the attraction to the target and repulsion from the defenders. We say that the synchronous intercept mission is accomplished, if the attacker robot is caught/destroyed by the defender robots synchronously in finite time, or the attacker robot is prevented from reaching the target for infinite time.

In particular, our paper makes three main contributions. First, we provide an effective formulation of the synchronous intercept in the defense-intrusion game, where the defender robots need to intercept the attacker robot synchronously. Second, the Attacker-oriented and Neutral-position-oriented intercept strategies are addressed for the defender robots to accomplish this synchronous intercept mission. These strategies task the defender robots with reaching either the attacker robot or a dynamic neutral position between the attacker robot and the target, respectively, while keeping a synchronous motion. Finally, we derive the feasible area for initially distributing defender robots so that they can accomplish a contact or a stable non-contact intercept and analyze the effectiveness and noise tolerance for each intercept strategy. The proposed strategies are of interest and can be extended for real intercept missions, such as territorial boundary defense with multiple autonomous robots, and aircraft escort with multiple autonomous vehicles.

\section{Related work}

The defense-intrusion game can be traced back to the pursuit-evasion game. Pursuer and evader in nature usually correspond to predator and prey, respectively. In an artificial world, they may be robotic vehicles. The solution of the single pursuer, single evader game is determined by construction of Apollonius circles which determine the dominance areas. For further definitions and basic notions, see Littlewood (1986); Basar and Olsder (1999).

An important subset of pursuit-evasion games is the two pursuers, single evader games. The objective of the pursuers is that at least one of them point-captures the evader, whereas the objective of the evader is to avoid or delay capture as long as possible. In this case, the time-optimal evading strategy (Makkapati et al, 2018) and conditions under which pursuit can be completed in the game (Bhattacharya and Hutchinson, 2008) were investigated. A particular pursuit-evasion game, where a faster evader attempts to pass the gap between two pursuers was addressed (Hagedorn and Breakwell, 1976; Zha et al, 2016). The authors considered the conditions under which the evader or pursuers can win the game, as well as a barrier that separates the state space into disjoint parts associated with each player's winning region. A three-player lifeline game was considered, in which a single evader is tasked with reaching a lifeline prior to capture (Zhang and Zha, 2018). They provided a resultant force method for the evader to balance the trade-off between reaching the lifeline and avoiding capture, as well as an escape zone for the evader.

The study of multiplayer pursuit-evasion games has been revitalized in recent years owing to the growing interest in general multi-agent system. In the multiple pursuers, single evader games, most of the literatures consider the conditions under which the pursuers can capture the evader, involving the minimum number and initial spatial distribution required as well as the cooperative strategies of the pursuers (Kopparty and Ravishankar, 2005; Turetsky, 2008; Oyler et al, 2016; Chen et al, 2016a; Zhou et al, 2016). For example, Alexander et al (2009) used simple tools from geometric convexity to obtain a necessary and sufficient condition for eventual capture in equal-speed discrete-time multi-pursuer 
capture games on convex Euclidean domains of arbitrary dimension and shape. Kothari et al (2014) proposed a simple capturing strategy for holonomic systems based on the idea of minimizing safe-reachable areas. Oyler et al (2016) provided the dominance regions in the presence and absence of obstacles. Zhou et al (2016) presented a decentralized control scheme for cooperative pursuit of a single evader based on minimizing the area of the generalized Voronoi partition of the evader in bounded, simply-connected planar domains.

In the single pursuer, multiple evaders games, Zhou et al (2012) presented an open-loop approach which provides guaranteed survival time of the evader team for all initial conditions, without the limitation that the pursuer must capture the evaders in a specific sequence. The open-loop approach is also used to a tracking game (Takei et al, 2014). A gradient-based approach under this open-loop formulation in the presence of a faster pursuer, a pursuer with uncertain position and evaders with limited turning rates were further studied in their later work (Liu et al, 2013a,b, 2014).

The multiple pursuers, multiple evaders games, also well known as group chase and escape are widely observed in nature. Kamimura and Ohira (2010) proposed a lattice model, in which the pursuers approach the nearest evader, while the evader tries to escape from being caught by the nearest pursuers on a two-dimensional lattice with periodic boundary conditions. By means of statistical methods, they found that there exists an optimal number of pursuers for a given number of evader to minimize the cost of catching all evaders. Since then, many modified versions of the group chase and escape model were proposed. The literatures contain a large body of work on how to enhance the catching efficiency, such as adding interaction among pursuers (keep enough space among pursuers) (Saito et al, 2016), adding lazy or fast pursuers (Iwama and Sato, 2012; Masuko et al, 2017), and using a collective chasing strategy (Janosov et al, 2017). The literatures also contain the study of aggregation in group chase and escape. The literatures show that the aggregation behavior can benefit the survivability of evaders owing to many eyes effect (Siegfried and Underhill, 1975), confusion and dilution effect (Neill and Cullen, 1974), and unity effect (Bertram, 1978).

An important variant of pursuit evasion game is defense intrusion game. The Target-Attacker-Defender (TAD) game (Boyell, 1976) is a such game that simulates a defense-intrusion interaction. Because of the nature of this problem, many different types of cooperation mechanisms in the target-defender team have been developed (Wang and Li, 2015; Zha et al, 2016; Shishika and Kumar, 2018; Liang et al, 2019; Pachter et al, 2019; Garcia et al, 2019; Sun et al, 2019). Liang et al (2019) constructed a barrier that separates the whole space into the winning region of the attacker robot and the winning region of the target-defender team by means of Apollonius circles. Shishika and Kumar (2018) considered a variant of TAD game termed the Perimeterdefense problem, where multiple defender robots defend a region's perimeter from attacker robots. The defender robots employed a decomposition method based on assignment (i.e., each defender robot is assigned to a unique attacker robot) as the team strategy. Most of the aforementioned studies focus on the optimal strategies and conditions for a team to dominate the game using a differential game approach. Another well known multi-player defense-intrusion interaction is reach-avoid game, where the attacking team attempts to reach a certain target location while the defenders aim to capture the attackers to prevent the attacking team from reaching its goal (Chen et al, 2014a,b, 2016b; Zhou et al, 2018).

Other literatures related to defense-intrusion game usually impose some specific scenarios. For example, Pan et al (2012) studied a particular defense-intrusion game with multiple pursuers, a single evader and a single defender, played in a convex domain with an exit through which the evader may escape. Another unconventional defense-intrusion game is referred to as Cooperative-Confinement-Escape (CCE) game, in which the defenders are moving on the circle with attempt to prevent possible escape of a single evader who is initially located inside the circle ( $\mathrm{Li}, 2016 \mathrm{~b}, \mathrm{a}$; Ramana and Kothari, 2017). The defense-intrusion game is also seen in multi-robot systems. Martin et al (2010) considered a multi-robot system with three different robots. The predator robot aims to capture the prey robot, while the defender robot protects the prey robot from the predator robot, but is also tasked with guiding the prey robot to the nest in a finite time. A reinforcement learning setup is presented to find an optimal policy to control the defender robot in its role of protecting the prey against the predator robot.

The remaining of this paper is arranged as follows: Section 3 describes the scenario of the synchronous intercept problem and two typical intercept patterns. Section 4 describes the motion dynamics of the defender robots and attacker robot. Section 5 proposes two kind$\mathrm{s}$ of synchronous intercept strategies corresponding to two typical intercept patterns. The simulation results and analysis are presented in Section 6. Finally, Section 7 concludes the paper with a brief summary. 


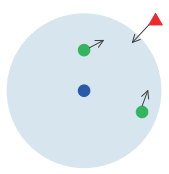

(a)

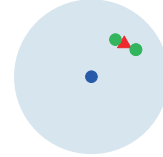

(b)

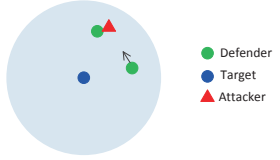

(c)
Fig. 1 (a) Illustration of the synchronous intercept problem with two defender robots. (b) Successful synchronous interception. (c) Failed synchronous interception.

\section{Problem Statement}

\subsection{Scenario of the synchronous intercept problem}

Consider two homogeneous defender robots and a single attacker robot in the 2-dimensional obstacle-free Euclidean space, with positions of $\mathbf{p}_{i}(t) \in \mathbb{R}^{2}(i=1,2)$ and $\mathbf{p}_{a}(t) \in \mathbb{R}^{2}$, and maximum speeds of $v_{d}$ and $v_{a}$, respectively. The defender robots attempt to protect a stationary target $\mathbf{p}_{0}$ against the attacker robot by a synchronous intercept, while the attacker robot attempts to reach/attack the target with avoidance to the defender robots, as illustrated in Fig. 1(a). The attacker robot can be caught/destroyed only when it is attacked by both defender robots synchronously (Fig. 1(b)), if either of the two defender robots reach the attacker robot independently, then it is destroyed by the attacker robot, leading to a failing intercept mission (Fig. 1(c)). No collision avoidance is considered in this paper. Let $R$ be the relative distance from the target to the attacker robot. The results of synchronous intercept can be represented as:

Definition 1 Synchronous intercept is failed if there exists $t^{*}$ such that $R\left(t^{*}\right) \leq \delta$, where $\delta>0$ is a small constant, denoting the size of attack area (security tolerance).

This definition presents that a failed synchronous intercept means the attacker robot reaches the target before it is caught by the defender robots.

Definition 2 Synchronous intercept is successful if there exists $t^{*}$ such that $\sum_{i=1}^{2} \varphi_{i}\left(t^{*}\right)=2$, or $R(t)>\delta$ holds for all $t \geq 0$, where $\varphi_{i}(t)$ denotes a Boolean function. $\varphi_{i}(t)=1$ means the defender robot $i$ reaches the attacker robot at time $t$, otherwise, $\varphi_{i}(t)=0$.

This definition presents that a successful synchronous intercept can be achieved by a synchronous attack or synchronous defense.

For synchronous attack, we give two constraints: synchronization constraint and shortest time constrain$t$.
Definition 3 Synchronization constraint is met if $\sum_{i=1}^{2} \varphi_{i}\left(t^{*}\right)=2$ where $t^{*}$ is the capture time.

The synchronization constraint requires that both defender robots must reach the attacker robot synchronously.

Definition 4 Shortest time constraint is met if $v_{2}(t)=$ $v_{d}$ at each movement, where $v_{2}(t)$ denotes the speed of the further defender (the defender robot 2 is assumed to be the further defender throughout the paper).

The shortest time constraint requires that the further defender robot must approach the attacker robot at its maximum speed.

\subsection{Two intercept patterns}

According to definition 2, a successful synchronous intercept can be achieved by synchronous attack or synchronous defense. Due to this fact, two intercept patterns: contact intercept pattern and non-contact intercept pattern can be designed with regards to synchronous attack and synchronous defense, respectively.

The contact intercept pattern mainly focus on the synchronous attack behavior in the synchronous intercept problem, where the defender robots attempt to reach/attack the attacker robot synchronously in finite time. This contact intercept pattern is observed in the animal kingdom. For example, hyenas display synchronous behavior both when hunting their prey and when performing anti-attack maneuvers on predators. Contact intercept patterns can also be applied to artificial systems. Missile interception is one such example, where the approaching missile must be destroyed before reaching a protected area.

The non-contact intercept pattern mainly focuses on synchronous defense behavior in the synchronous intercept problem. This behavior sees the defender robots prevent the intrusion indefinitely, so that the attacker robot can never reach/attack the target. It is worth noting that the defender robots are also unable to destroy the attacker robot. In the animal kingdom, a lioness displays a non-contact intercept pattern when attempting to protect her cubs against a predator or predators, where the primary objective of the lioness is to keep the attacker away from its cubs. In artificial system, destruction of every suspected attacker may be excessive or risky, especially if misidentification as an attacker is possible. For example, the goal may be to protect a sensitive area from being disturbed, therefore expelling a suspected attacker would be a more reasonable measure than destroying them. 
Our goal is to design synchronous intercept strategies for the two intercept patterns, such that the defender robots can perform the synchronous intercept mission according to practical needs. The proposed approach is not considering collision avoidance.

\section{The model}

Assume that the instantaneous positions of the players are known to all agents. The attacker robot is an intelligent agent with the ability to execute defender avoidance (the attacker robot is assumed to move with a constant velocity on a straight path to the target as in Shishika and Paley (2019)). When $v_{d}<v_{a}$, the attacker robot can always win the game by combining this advantage with defender avoidance to reach the target. Therefore, we consider only the case of $v_{d} \geq v_{a}$, and use $\gamma=v_{d} / v_{a}$ to denote the speed ratio, hence $\gamma \geq 1$.

\subsection{The attacker robot}

The position and velocity of the attacker robot are updated as:

$\mathbf{p}_{a}(t+\Delta t)=\mathbf{p}_{a}(t)+\mathbf{v}_{a}(t) \Delta t$

$\mathbf{v}_{a}(t)=v_{a} \hat{\mathbf{d}}_{a}(t)$

where $v_{a}$ is a scalar denoting the speed of the attacker robot, $\hat{\mathbf{d}}_{a}(t)$ is a unit vector denoting the desired direction in the next step, $\Delta t$ is the length of one time step. The driving forces are simulated by a virtual attractive force from the target and a virtual repulsive force from each defender robot, the maneuvering mode of the attacker robot is given as

$\mathbf{d}_{a}=-k_{r} \sum_{i=1}^{2} \frac{1}{\left\|\mathbf{p}_{i}-\mathbf{p}_{a}\right\|^{\alpha}} \hat{\mathbf{p}}_{i a}+k_{a} \frac{1}{\left\|\mathbf{p}_{0}-\mathbf{p}_{a}\right\|^{\alpha}} \hat{\mathbf{p}}_{0 a}$

where $\left\|\mathbf{p}_{i}-\mathbf{p}_{a}\right\|$ and $\left\|\mathbf{p}_{0}-\mathbf{p}_{a}\right\|$ are distances from $\mathbf{p}_{a}$ to $\mathbf{p}_{i}$ and $\mathbf{p}_{a}$ to $\mathbf{p}_{0}$ respectively. $\alpha \geq 0$ is the exponent of distance, adjusting the effect of distance on the corresponding driving force, e.g. $\alpha=0$ means the attractive/repulsive force is distance-independent. $\hat{\mathbf{p}}_{i a}$ and $\hat{\mathbf{p}}_{0 a}$ are unit vectors from $\mathbf{p}_{a}$ to $\mathbf{p}_{i}$ and $\mathbf{p}_{a}$ to $\mathbf{p}_{0}$ respectively. $k_{r}>0$ and $k_{a}>0$ are the repulsive and attractive coefficients, respectively. $k_{a} / k_{r}$ describes the tradeoff between approaching the target and avoiding the defender robots, where together with $\alpha$ these variables determine the attacker's invasion strategy. For example, when $k_{a} / k_{r} \rightarrow \infty$ (i.e. $k_{r} \rightarrow 0$ ), the attacker robot attacks the target directly without attempting to avoid any defender robots; when $k_{a} / k_{r} \rightarrow 0$ (i.e. $k_{a} \rightarrow 0$ ), the attacker robot attempts to escape from the defender robots with no attraction to the target. Unless otherwise stated, both repulsion and attraction increase as the distance between agents is reduced $(\alpha=1)$. Therefore, the nearest defender robot has the most influence over the attacker's behaviour and the closer the attacker robot is to the target the greater the attractive force.

\subsection{The defender robot}

The position and velocity of each defender robot are updated as

$\mathbf{p}_{i}(t+\Delta t)=\mathbf{p}_{i}(t)+\mathbf{v}_{i}(t) \Delta t$

$\mathbf{v}_{i}(t)=\frac{\mathbf{p}_{i}(t+\Delta t)-\mathbf{p}_{i}(t)}{\Delta t}$

where $\mathbf{v}_{i}(t)$ is the control of defender robot $i$. $\mathbf{p}^{*}{ }_{i}(t+\Delta t)$ is the expected position that can be obtained via the intercept strategy described by optimization formulation as

$$
\begin{array}{ll}
\min & f_{i}\left(\mathbf{p}^{*}{ }_{i}(t+\Delta t), \mathbf{p}_{i}(t), \mathbf{p}_{a}(t), \mathbf{p}_{0}\right), \quad i=1,2 \\
\text { w.r.t. } & \mathbf{p}^{*}{ }_{i}=\left[p_{i x}^{*}, p_{i y}^{*}\right] \\
\text { s.t. } & v_{i}(t) \leq v_{d}
\end{array}
$$

where $v_{d}$ is the maximum speed of the defender robot (two defender robots are assumed to have the same maximum speed).

The intercept strategy functions $f$ consider the current positions of the target, the attacker robot and the defender robots. The specific form of the function is dependent on the two intercept patterns, which are detailed with the optimization formulation in the next section.

\section{Intercept Strategy}

In this section, we first illustrate why an intercept strategy is beneficial in the synchronous intercept problem by analyzing the efficiency of two reference strategies. Two attacker-oriented strategies for the synchronous attack mission with regards to contact intercept pattern are then proposed, following by four versions of neutralposition-oriented strategies with regards to non-contact intercept pattern.

\subsection{Why intercept strategy is needed}

The Selfish strategy was introduced in Kamimura and Ohira (2010), where each defender robot makes a maximal advance towards the attacker robot during each movement, see Fig. 2(a). This strategy reduces each 


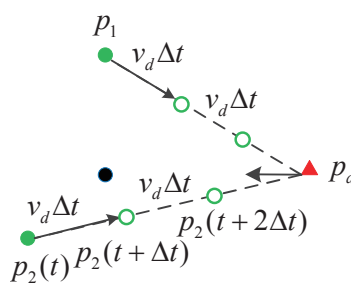

(a)

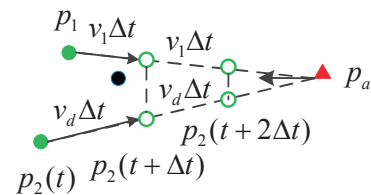

(c)

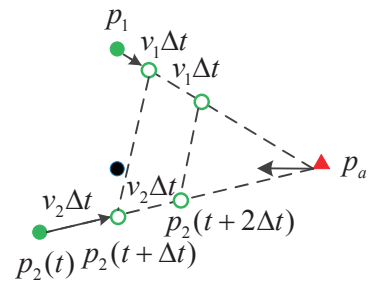

(b)

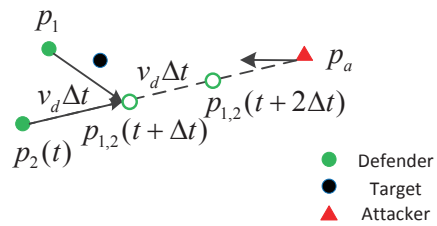

(d)

Fig. 2 (a) Selfish strategy. (b) Cone strategy. (c) Parallel strategy. (d) Aggregation strategy. The defense-intrusion game should be dynamic for both defender and intruder robots. For clarity, here the intercept strategies are presented with changing positions of the defender robots and stationary intruder robot.

defender's distance to the attacker independently, but it does not ensure that both defender robots reach the attacker robot synchronously and so cannot guarantee the synchronization constraint.

\section{Selfish strategy}

$\min f_{i}=\left\|\mathbf{p}^{*}{ }_{i}(t+\Delta t)-\mathbf{p}_{a}(t)\right\|^{2}, \quad i=1,2$

w.r.t. $\quad \mathbf{p}^{*}{ }_{i}=\left[p_{i x}^{*}, p_{i y}^{*}\right]$

s.t. $\quad v_{i}(t) \leq v_{d}$

The Cone strategy, proposed in Bopardikar and Suri (2014), is regarded as another reference strategy, in which each defender robot tries to minimize the distance from the attacker robot subject to the constraint that both defender robots are the same distance from the attacker robot, see Fig. 2(b) (one can refer to Bopardikar and Suri (2014) for a more precise expression for the new locations in a Cone move). The synchronization constraint can be guaranteed but the shortest time constraint cannot, as it does not ensure that the further defender robot maximally advances towards the attacker robot during each movement.

Cone strategy

$$
\begin{array}{ll}
\min & f_{i}=\left\|\mathbf{p}_{i}(t+\Delta t)-\mathbf{p}_{a}(t)\right\|^{2} \\
& +\left(\left\|\mathbf{p}_{i}(t+\Delta t)-\mathbf{p}_{a}(t)\right\|-\left\|\mathbf{p}_{j}(t)-\mathbf{p}_{a}(t)\right\|\right)^{2} \\
\text { w.r.t. } & \mathbf{p}_{i}^{*}=\left[p_{i x}^{*}, p_{i y}^{*}\right] \\
\text { s.t. } & v_{i}(t) \leq v_{d}, \quad i, j=1,2, \quad i \neq j
\end{array}
$$

The synchronization constraint and shortest time constraint are not simultaneously satisfied by the above two strategies, which results in a sub-optimal synchronous intercept mission. Therefore, more efficient intercept strategies for the synchronous intercept mission shall be proposed.

\subsection{Attacker-oriented strategy}

Attacker-oriented strategy is defined as an attack behavior that the defender robots aim to destroy the attacker robot by reaching the position of the attacker robot synchronously. We propose two Attacker-oriented intercept strategies with the constraints of synchronization and shortest time so that the defender robots rapidly approach the attacker robot while maintaining synchronization.

The Parallel strategy shall be described first, in which each defender robot tries to reach the attacker robot as early as possible while keeping parallel with the other one, as illustrated in Fig. 2(c). In other word$\mathrm{s}$, the further defender robot approaches the attacker robot at its maximum speed, while the closer defender robot reduce its speed in proportion to the proximity to the attacker robot. A parallel movement then appears from the exist of velocity difference.

\section{Parallel strategy}

$$
\begin{aligned}
& \min \quad f_{i}=\left\|\mathbf{p}_{i}{ }_{i}(t+\Delta t)-\mathbf{p}_{a}(t)\right\|^{2} \\
& \text { w.r.t. } \quad \mathbf{p}^{*}{ }_{i}=\left[p_{i x}^{*}, p_{i y}^{*}\right] \\
& \text { s.t. } \quad v_{i}(t)=v_{d} \cdot \min \left\{\frac{\left\|\mathbf{p}_{i}(t)-\mathbf{p}_{a}(t)\right\|}{\left\|\mathbf{p}_{2}(t)-\mathbf{p}_{a}(t)\right\|}, 1\right\}, \quad i=1,2
\end{aligned}
$$


The constraints of synchronization and shortest time can be guaranteed by using this strategy. The shortest time is ensured by allowing the further defender robot to advance maximally towards the attacker robot during each movement. The synchronization constraint is ensured by the closer defender robot, to the attacker robot, maintaining a parallel advance.

The second strategy is Aggregation, in which the defender robots aggregate first and then approach the attacker robot together, as illustrated in Fig. 2(d). Before the aggregation, the constraint $h_{1}$ ensures that the meeting point is located at the segment between the further defender robot and the attacker robot, and the constraint $h_{2}$ ensures that the distances from each defender robot to the meeting point are equal.

Aggregation strategy

Before aggregation

$\min f_{i}=\left\|\mathbf{p}_{i}{ }_{i}(t+\Delta t)-\mathbf{p}_{m}(t)\right\|^{2}$,

w.r.t. $\quad \mathbf{p}^{*}{ }_{i}=\left[p_{i x}^{*}, p_{i y}^{*}\right]$

s.t. $h_{1}: \mathbf{p}_{m} \in \mathbf{p}_{2} \mathbf{p}_{a}$

$h_{2}:\left\|\mathbf{p}_{i}-\mathbf{p}_{m}\right\|=\left\|\mathbf{p}_{2}-\mathbf{p}_{m}\right\|$

$v_{i}(t)=v_{d}, \quad i=1,2$

After aggregation

$\min f_{i}=\left\|\mathbf{p}^{*}{ }_{i}(t+\Delta t)-\mathbf{p}_{a}(t)\right\|^{2}$

w.r.t. $\quad \mathbf{p}^{*}{ }_{i}=\left[p_{i x}^{*}, p_{i y}^{*}\right]$

s.t. $\quad v_{i}(t)=v_{d}, \quad i=1,2$

where $\mathbf{p}_{m}$ is the meeting point. The constraints of synchronization and shortest time can also be guaranteed by using this strategy. The synchronization constraint is ensured by the aggregation behavior and the shortest time constraint is ensured by allowing the further defender robot to advance at its maximum velocity towards the attacker robot during each movement.

We further discuss how to get the meeting point $\mathbf{p}_{m}$ for arbitrary initial positions of the defenders. Assume that the attacker robot, the closer defender robot and the further defender robot are initially placed at coordinates $\mathbf{p}_{a}(0,0), \mathbf{p}_{1}(x, y)$ and $\mathbf{p}_{2}(R, 0)$ respectively (see Fig. 3).

Assume the meeting point is $\mathbf{p}_{m}(z, 0)$. The constraint $h_{2}$ in Eq. (10) can be described as

$R-z=\sqrt{(x-z)^{2}+y^{2}}$

from which, we have

$z=\frac{R^{2}-\left(x^{2}+y^{2}\right)}{2(R-x)}$

Since the robot 1 is the closer defender, thus $x^{2}+$ $y^{2} \leq R^{2}$. From this point, we can get $0 \leq z \leq R$ from Eq. (13), which means there must be a meeting point

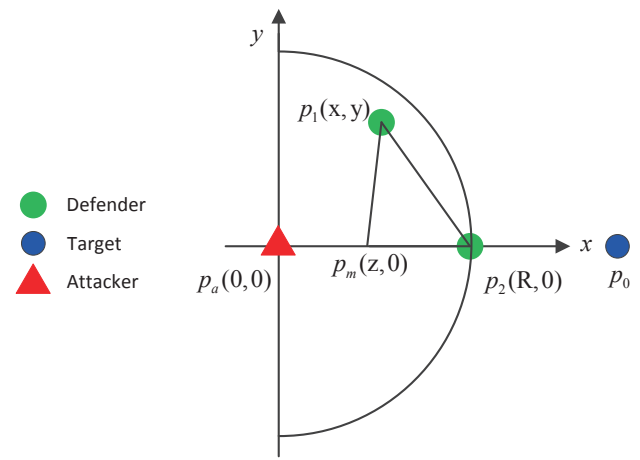

Fig. 3 Illustration of how to get the meeting point.

that is located at the segment between $\mathbf{p}_{2}$ and $\mathbf{p}_{a}$ for arbitrary initial positions of the defenders. For example, (1) when $x=0, y=R, \mathbf{p}_{m}(0,0)$; (2) when $x=0, y=0$, $\mathbf{p}_{m}(R / 2,0)$; (3) when $x=R, y=0, \mathbf{p}_{m}(R, 0)$.

\subsection{Neutral-position-oriented strategy}

Neutral-position-oriented strategy is defined as a defense behavior that the defender robots aim to prevent the target from reaching the target infinitely. Capitalizing on the knowledge of the attacker's intends to reach the target, the defender robots can approach a neutral position between the attacker robot and the target, so that the attack and defense behaviors can both be considered. From this idea, we propose another group of intercept strategies referred to as Neutral-positionoriented strategies that generate a non-contact intercept pattern. The neutral position $\mathbf{p}_{m}$ is obtained by

$\mathbf{p}_{m}(t)=(1-\lambda(t)) \mathbf{p}_{0}+\lambda(t) \mathbf{p}_{a}(t)$

$\lambda(t)=\frac{1}{n} \sum_{i=1}^{n}\left(\frac{\left\|\mathbf{p}_{i}(t)-\mathbf{p}_{a}(t)\right\|}{\left\|\mathbf{p}_{i}(0)-\mathbf{p}_{a}(0)\right\|}\right)$

where $n$ is the number of defender robots $(n=2$ in this paper). $\lambda \in[0,1]$ is the transferred parameter, determining the defender's expected position between the target and the attacker robot. When $\lambda \rightarrow 0, \mathbf{p}_{m}(t) \rightarrow$ $\mathbf{p}_{0}$ and the Neutral-position-oriented strategy degenerates into a defense-only intercept strategy; When $\lambda \rightarrow$ $1, \mathbf{p}_{m}(t) \rightarrow \mathbf{p}_{a}$ and the Neutral-position-oriented strategy degenerates into an attack-only intercept strategy (i.e. Attacker-oriented strategy). Since $\lambda$ increases from 0 to 1 with the defense-intrusion process, the expected neutral position will transfer from $\mathbf{p}_{0}$ to $\mathbf{p}_{a}$ continuously, as illustrated in Fig. 4.

The approaching position $\mathbf{p}_{a}$ can be replaced with the Neutral Position (NP) $\mathbf{p}_{m}$ for the Attacker-oriented strategies, described previously. The NP versions of these 


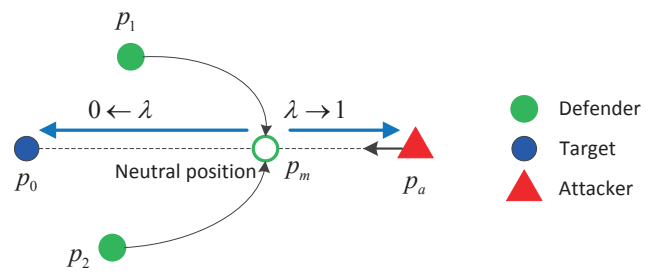

Fig. 4 Illustration of Neutral-position-oriented strategy.

strategies are known as the Selfish-NP, Cone-NP, Parallel$N P$ and Aggregation-NP strategy. For example, Selfish$N P$ strategy can be written as:

$\min f_{i}=\left\|\mathbf{p}^{*}{ }_{i}(t+\Delta t)-\mathbf{p}_{m}(t)\right\|^{2}, \quad i=1,2$ w.r.t. $\quad \mathbf{p}^{*}{ }_{i}=\left[p_{i x}^{*}, p_{i y}^{*}\right]$

s.t. $v_{i}(t) \leq v_{d}$

The neutral position $\mathbf{p}_{m}$ is always located at the segment between the target and the attacker robot, this should facilitate defensive behavior. However, this does not ensure that defender robots synchronously catch the attacker robot, but rather improves their ability to prevent the attacker robot from reaching the target for infinite time.

\section{Results}

Without loss of generality, the target is a stationary point at the origin and the attacker robot is initially placed at $(100,0)$. Unless otherwise stated, the following parameter values are $\delta=0.1, \alpha=1$ and $k_{a} / k_{r}=10$.

\subsection{Feasible area of initial distribution}

Definition 5 The area is defined as feasible area if the defender robots which are initially located in this area can lead to a successful contact intercept pattern or stable non-contact intercept pattern.

In this section, we investigate the feasible area corresponding to the Attacker-oriented strategy and the Neutral-position-oriented strategy.

\subsubsection{Attacker-oriented strategy}

By using the Attacker-oriented strategy, for $\gamma>1$, a feasible area of initial distribution exists, where the defender robots can accomplish the contact intercept if initially deployed inside the area. The relative distance $d$ from the attacker robot to the farthest defender robot, and the line-of-sight angle $\theta$ (see Fig. 5) can be derived as follows:

$\dot{d}=-v_{a} \cos (\theta)-v_{d}, \quad d\left(t_{0}\right)=d_{0}$

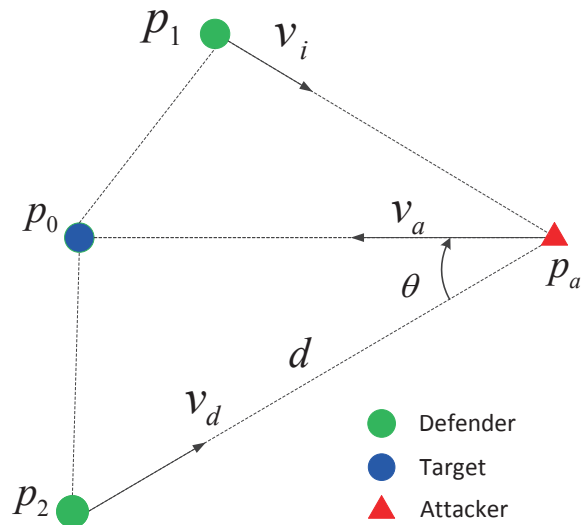

Fig. 5 Illustration of relative distance and line-of-sight angle.

$\dot{\theta}=\frac{1}{d} v_{a} \sin (\theta), \quad \theta\left(t_{0}\right)=\theta_{0}$

According to the definition of successful intercept, a general synchronous attack should take place before the attacker robot reaches the target. The critical condition for a successful intercept is that the synchronous attack event happens just as the attacker robot arrives at the target position, which can be characterized by

$d\left(t^{*}\right) \leq \delta$

$t^{*}=\frac{R_{0}-\delta}{v_{a}}$

where $\delta>0$ is a small constant, denoting the size of attack area (security tolerance), $R_{0}$ is the initial distance from the attacker robot to the target, $v_{a}$ is the maximum speed of the attacker robot and $t^{*}$ denotes the shortest time for the attacker robot to reach the target. The initial distribution of the farthest defender robot is described by $\left(d_{0}, \theta_{0}\right)$, which satisfies the critical condition that then determines the feasible area. As the feasible area is merely determined by the farthest defender, we can obtain the similar feasible area when the number of defender robots is greater than 2 .

There is no analytical solution for the differential equations Eq. (17) and Eq. (18), with numerical solutions obtained using MATLAB. The feasible area, associated with the intercept trace, is shown in Fig. 6(a). The "ellipse" boundary separates the state space into two disjoint areas: feasible area and infeasible area for initial defender deployment.

There is no analytical solution for the "semi-minor axis" of the feasible area. However, we can analytically derive the "semi-major axis" of the boundary by deploying the attacker, target and defender robot in a line, see Fig. 7(a). In this case, the critical condition is that the attacker robot reaches (or nearly reaches) the 


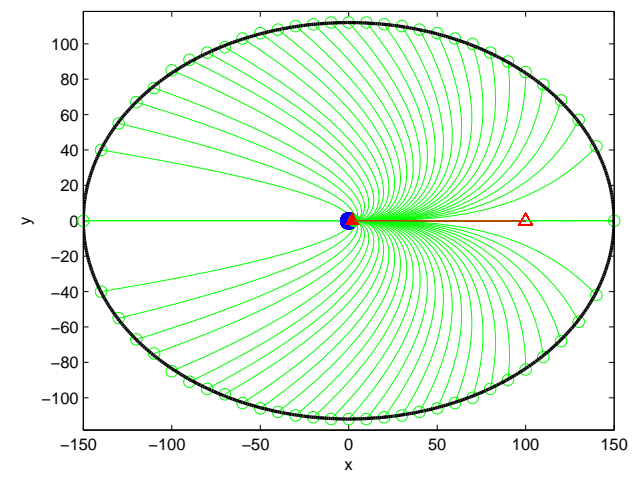

(a)

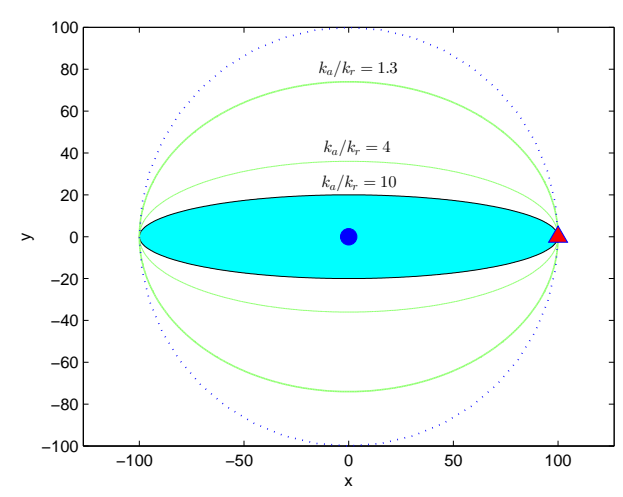

(b)

Fig. 6 The feasible area of initial distribution by using Attacker-oriented strategy. (a) $\gamma=1.5, L=150, k_{a} / k_{r}=10$. (b) $\gamma=1, L=100, k_{a} / k_{r}=10$. The attacker robot is initially placed at $(100,0)$, marked with a red triangle.

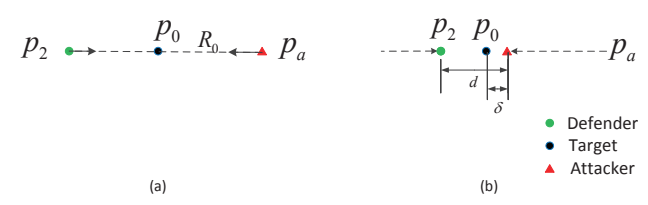

Fig. 7 (a) The collinear distribution of defender, target and attacker. (b) The critical condition. $\mathbf{p}_{2}$ is the further defender robot.

target when the repulsive force is equal to the attractive force, see Fig. 7(b). The critical condition can be characterized as

$n \frac{k_{r}}{d^{\alpha}}=\frac{k_{a}}{\delta^{\alpha}}$

where $k_{r}$ and $k_{a}$ are repulsion and attraction coefficients, respectively. $\alpha$ is the distance exponent, describing the effect of distance on the corresponding driving force. From Eq. (21), we can get

$d=\sqrt[\alpha]{\frac{n k_{r}}{k_{a}}} \delta$

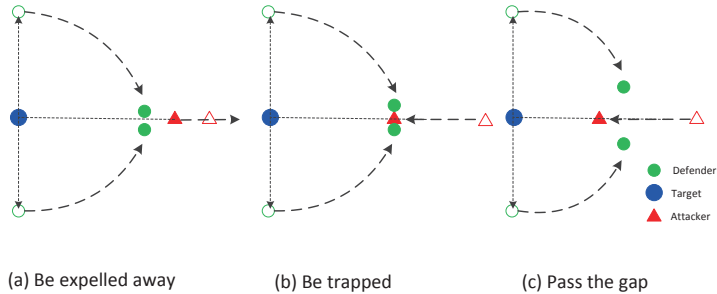

Fig. 8 Three cases under the symmetrical distribution.

The "semi-major axis" of the boundary can then be obtained as

$L=\left(\sqrt[\alpha]{\frac{n k_{r}}{k_{a}}} \delta-\delta\right)+v_{d}\left(\frac{R_{0}-\delta}{v_{a}}\right)$

where $R_{0}$ is the initial distance between the attacker robot and the target, and $\frac{R_{0}-\delta}{v_{a}}$ is the time that the attacker robot spent on reaching the equilibrium point. If the parameter $\delta$ is infinitely small, then the "semimajor axis" is merely determined by $\gamma$ and $R_{0}$ as

$L=\gamma R_{0}$

For $\gamma=1$, a feasible area exists with a special constraint, in which the defender robots must be deployed symmetrically about the line between the target and the attacker robot inside the feasible area. This special distribution constraint can be explained as follows. In most situations, the attacker robot cannot be caught by the defender robots when they have the same maximum velocity, due to the attacker's capture avoidance behavior. But by deploying the defender robots symmetrically, the attacker robot is either expelled, trapped, or able to pass through a gap and reach the target, as detailed in Fig. 8. If we consider the situations where the attacker robot is expelled away or trapped as successful cases, then we can get the feasible area with a symmetrical distribution constraint. This feasible area is shown in Fig. 6(b), where it can be seen that a strategy with a bigger $k_{a} / k_{r}$ ratio requires a more narrow feasible area to be successful.

The capture time upper bound is obtained when the defender robots are deployed on the boundary of the feasible area, while the defender robots insider the feasible area can synchronously capture the attacker within a shorter time. For all situations of boundary distribution of defender robots, we can get the same capture time upper bound as

$t_{\max }^{*}=\frac{L}{v_{d}}=\frac{R_{0}}{v_{a}}$

where $L=\gamma R_{0}$, and the parameter $\delta$ is assumed to be infinitely small. 


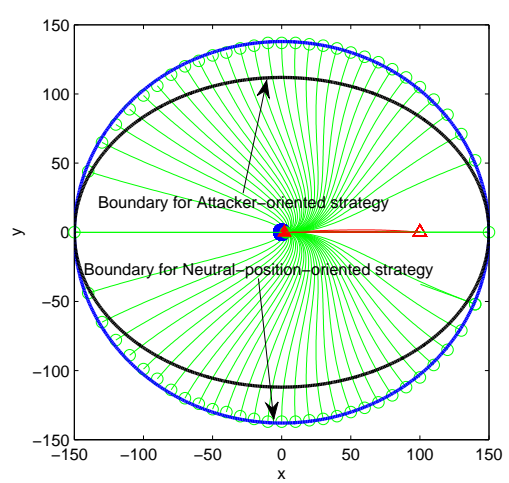

(a)

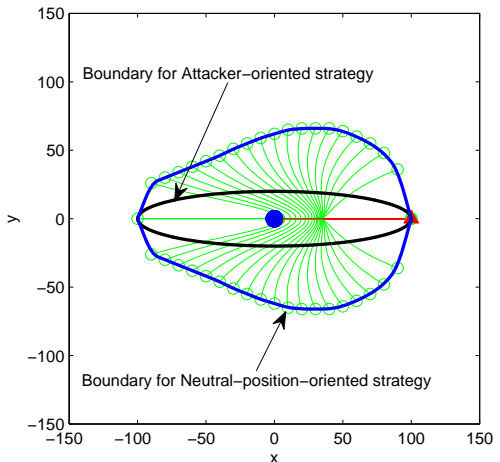

(b)

Fig. 9 The feasible area of initial distribution by using Neutral-position-oriented strategy. (a) $v_{d}=1.5 v_{a}, L=150, k_{a} / k_{r}=10$. (b) $v_{d}=v_{a}, L=100, k_{a} / k_{r}=10$. The attacker robot is initially placed at $(100,0)$, marked with a red triangle.

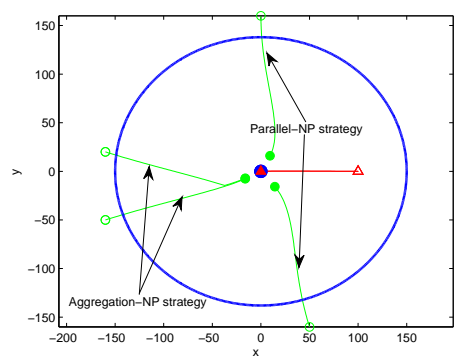

(a) Outside the feasible area $(\gamma=1.5)$

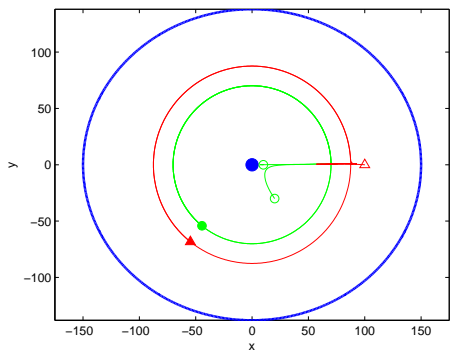

(d) Parallel-NP strategy $(\gamma=1.5)$

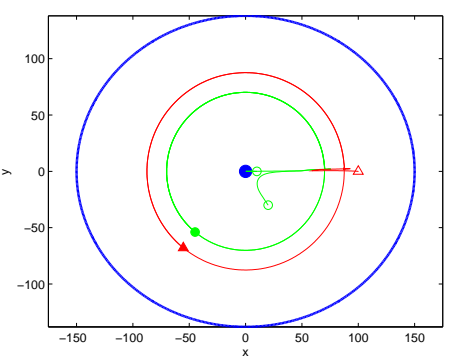

(b) Selfish-NP strategy $(\gamma=1.5)$

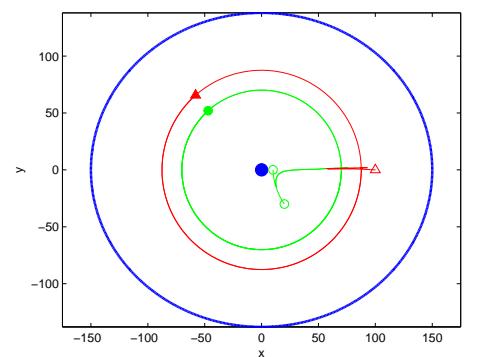

(e) Aggregation-NP strategy $(\gamma=1.5)$

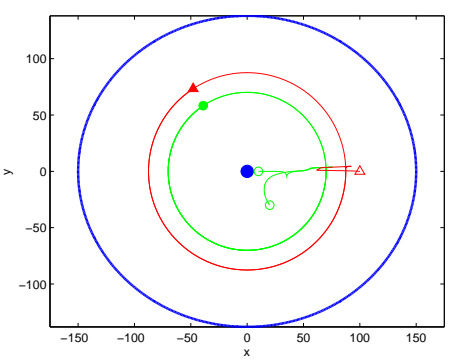

(c) Cone-NP strategy $(\gamma=1.5)$

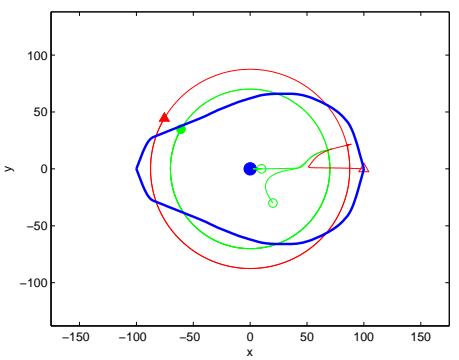

(f) Selfish-NP strategy $(\gamma=1)$

Fig. 10 Verification of feasible area and stable non-contact intercept pattern. (a) Both Parallel-NP strategy and AggregationNP strategy cannot generate the stable non-contact intercept pattern if the defender robots are initially placed outside the feasible area. (b)-(e) If the defender robots are initially placed insider the feasible area, the stable non-contact intercept pattern occurs regardless of which Neutral-position-oriented strategy is used. (f) For the special case of $\gamma=1$, even the Selfish-NP strategy can generate a stable non-contact intercept pattern.

\subsubsection{Neutral-position-oriented strategy}

By using the Neutral-position-oriented strategy, for $\gamma \geq$ 1 , a feasible area of initial defender distribution exists for a stable non-contact pattern (there is no symmetrical distribution constraint for the situation of $\gamma=1$, we will elaborate on this point later). There is no analytical solution for the feasible area, the numerical solutions are again obtained via MATLAB. The feasible area, using Neutral-position-oriented strategy, is compared with the corresponding feasible area with the Attackeroriented strategy as shown in Fig. 9 (throughout the paper the solid black-colored lines correspond to the feasible area for Attacker-oriented strategy and blue-colored lines correspond to Neutral-position-oriented strategy). The results show that, for both $\gamma>1$ and $\gamma=1$ situations, the Neutral-position-oriented strategy allows a larger feasible area than that of the Attacker-oriented strategy. This larger area is a result of the neutral po- 
sition being closer to the defender robots, for any given scenario, than the position of the attacker robot.

By using the Neutral-position-oriented strategy, the system generates a stable periodic attractor, i.e. a stable non-contact intercept pattern. Fig. 10(a) shows that if the defender robots are initially placed outside of the feasible area, the Neutral-position-oriented strategy cannot generate a stable non-contact intercept pattern. Fig. 10(b)-(f) shows that if the defender robots are initially placed inside the feasible area, then any Neutral-position-oriented strategy can generate the periodic attractor. The results also show that the stable motion radii of the defender and the attacker robots are strategy independent, when given the same initial distribution. The mechanism behind this can be explained as follows.

The stable non-contact intercept pattern can be characterized by

$k_{a} \frac{1}{R^{\alpha}}=n k_{r} \frac{1}{d^{\alpha}}$

$(1-\lambda) R=r$

$\lambda=\frac{d}{n} \sum_{i=1}^{n} \frac{1}{d_{i}}$

where $r>0$ and $R>r>0$ denote the stable motion radii of the defender and attacker robots respectively. $d$ is the stable distance between the defender robot and the attacker robot $(d=R-r)$. $\alpha$ is the distance exponent, describing the effect of distance on the corresponding driving forces. $d_{i}$ is the initial distance from the defender robot $i$ to the attacker robot. Eq. (26) is derived from Eq. (3), and it holds since attractive and repulsive forces are balanced in the stable state. Eq. (27) is derived from Eq. (14), and it holds since the final neutral position is located at the defender's circle. Eq. (28) is derived from Eq. (15), and it holds since the distance between the defender robot and the attacker robot is a constant in the stable state, see Fig. 11.

Substitute Eq. (28) to Eq. (27), we get

$R=\frac{n}{\sum_{i=1}^{n} \frac{1}{d_{i}}}$

Substitute Eq. (29) to Eq. (26), we get

$d=\frac{n}{\sum_{i=1}^{n} \frac{1}{d_{i}}} \sqrt[\alpha]{\frac{n k_{r}}{k_{a}}}$

As $d=R-r$, we then have

$r=\frac{n}{\sum_{i=1}^{n} \frac{1}{d_{i}}}\left(1-\sqrt[\alpha]{\frac{n k_{r}}{k_{a}}}\right)$

It is found that the stable motion radius of the attacker robot is not affected by invasion strategies. It is

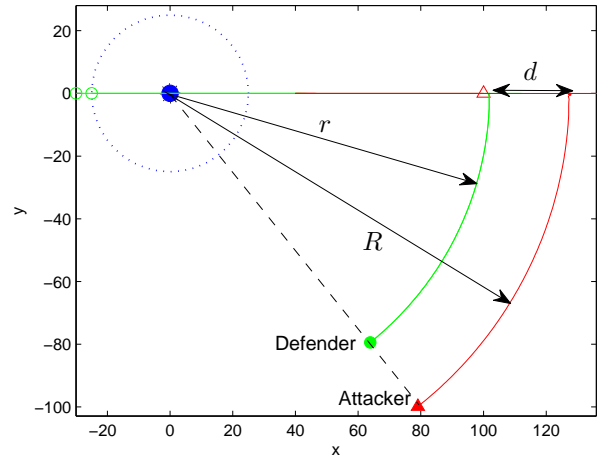

Fig. 11 Illustration of the stable non-contact intercept pattern (periodic attractor).

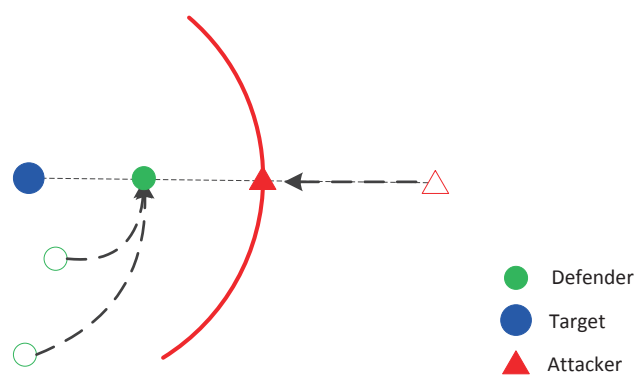

Fig. 12 Illustration of the asymmetrical distribution when using the Neutral-position-oriented strategy.

merely determined by the initial distribution and the number of defender robots. The stable motion radius of the defender robot is determined by many factors. As special cases, (1) when $n k_{r}=k_{a}$, then $r=0$, it means the non-contact intercept pattern is generated with all defender robots being located at the target; (2) when $k_{r} \rightarrow 0$, then $r \rightarrow R$, it means the stable motion radius of the defender robot increases as $k_{r}$ decreases, but no more than the stable motion radius of the attacker robot.

Fig. 10(f) also shows that the symmetrical distribution of defender robots is not necessary for the situation of $\gamma=1$ when using the Neutral-position-oriented strategy. Here we discuss why the defender robots do not have to be symmetrical. When using the Neutralposition-oriented strategy, the defender robots try to reach a neutral position between the target and the attacker robot, rather than the attacker robot. Hence, for both the symmetrical and asymmetrical distribution, a meeting point always appears on the path from the attacker robot to the target, as illustrated in Fig. 12. A stable non-contact intercept pattern is then generated for any $\gamma \geq 1$ in feasible area. 
Table 1 The statistical results of synchronous intercept, using Attacker-oriented strategies, where the strategy success and game duration are reported as $\left(\rho, \tau, \tau_{\max }\right)$.

\begin{tabular}{ccc}
\hline Strategy & $\gamma=1.5$ & $\gamma=1$ \\
\hline Selfish & $(0,51,56)$ & $(0,121,123)$ \\
Cone & $(1,61,71)$ & $(0,122,124)$ \\
Parallel & $(1,55,63)$ & $(0,123,126)$ \\
Aggregation & $(1,54,61)$ & $(0,121,124)$ \\
\hline
\end{tabular}

\subsection{Efficiency of the proposed intercept strategy}

For the synchronous intercept problem, the success rate $\rho$, the average intercept time $\tau$ and the worst case intercept time $\tau_{\max }$ are defined as

$\rho=\frac{N_{\text {suc }}}{N_{\text {tot }}}$

$\tau=\frac{\sum_{i=1}^{N_{\text {tot }}} t_{i}^{*}}{N_{\text {tot }}}$

$\tau_{\max }=t_{\max }^{*}$

where $N_{\text {suc }}$ is the number of successful cases out of $N_{\text {tot }}$ runs. $t_{i}^{*}$ is the intercept time for each simulation and $t_{\text {max }}^{*}$ is the worst case intercept time out of $N_{\text {tot }}$ runs. Now we use Monte Carlo simulations to investigate the efficiency of different intercept strategies. The defender robots are initially placed inside a circle, centered on the target, with a radius of 20 (this is a feasible area for all the following simulations) and the attacker robot is initially placed at $(100,0)$.

The results are obtained by 1000 simulations $\left(N_{\text {tot }}=\right.$ 1000). Tab. 1 shows the results of synchronous intercep$\mathrm{t}$ by using the Attacker-oriented strategy. For $\gamma=1.5$, the results show that the Selfish strategy cannot ensure a successful intercept $(\rho=0)$, while the other three strategies can ensure a successful intercept $(\rho=1)$. Compared to the proposed Parallel strategy and Aggregation strategy, Cone strategy needs more time, $\tau$ and $\tau_{\max }$ to achieve the synchronous intercept mission, which is consistent with the statement that the shortest time constraint cannot be guaranteed by this strategy. For $\gamma=1$, the synchronous intercept mission cannot be accomplished regardless of which Attacker-oriented strategy is used, because the defender robots are not deployed in a symmetrical distribution.

Tab. 2 shows the results of the synchronous intercept game, using Neutral-position-oriented strategy. For both $\gamma=1.5$ and $\gamma=1$, every Neutral-position-oriented strategy can ensure a successful defense $(\rho=1)$ with defense time $\tau=\tau_{\max }=1000$ (the longest simulation time is set by 1000 timesteps). Actually a stable state
Table 2 The statistical results of synchronous defense, using Neutral-position-oriented strategies, where the strategy success and game duration are reported as $\left(\rho, \tau, \tau_{\max }\right)$.

\begin{tabular}{ccc}
\hline Strategy & $\gamma=1.5$ & $\gamma=1$ \\
\hline Selfish-NP & $(1,1000,1000)$ & $(1,1000,1000)$ \\
Cone-NP & $(1,1000,1000)$ & $(1,1000,1000)$ \\
Parallel-NP & $(1,1000,1000)$ & $(1,1000,1000)$ \\
Aggregation-NP & $(1,1000,1000)$ & $(1,1000,1000)$ \\
\hline
\end{tabular}

(the stable non-contact intercept pattern) occurs during each simulation, where the defender robots and attacker robot orbit the target at the same angular speed with the paths forming two concentric circles.

\subsection{Effect of noise}

To evaluate the performance of the proposed intercep$\mathrm{t}$ strategies in real systems, noise is introduced as an external disturbance. Assume that the external disturbance affects the moving direction of the defender robots. The effect of external disturbance, $\mathfrak{R}_{\eta}\left[\mathbf{v}_{i}(t)\right]$, is simulated as a rotational disturbance that alters the desired direction of the defender robot using a random angle with a uniform distribution in the interval $[-\eta \pi, \eta \pi]$, where $\eta \in[0,1]$ is the noise strength.

The intercept results, for different noise strength$\mathrm{s}$, are shown in Fig. 13. The results presented show that the Selfish strategy can barely accomplish the synchronous intercept mission, regardless of noise strength. The other three Attacker-oriented strategies have a good tolerance for noise until $\eta<0.3$, after which the defender robots lose their ability to accomplish synchronous intercept quickly, see Fig. 13(a). For $\gamma=1$, the defender robots can barely accomplish the defense mission, even in the absence of noise, therefore the curve of success rate changing with noise strength in this case is omitted. Fig. 13(b) shows that by using Neutral-positionoriented strategy, if the defender robots have a speed advantage over the attacker robot $(\gamma=1.5)$, then they have a better tolerance for noise $(\eta \leq 0.4$ for $\gamma=1.5$ and $\eta \leq 0.1$ for $\gamma=1$ ). Fig. 14 illustrates two successful intercept patterns when noise is present.

In order to further investigate the influence of the speed ratio $\gamma$ in the presence of noise, we define the maximum tolerable noise $\eta^{*}$ as the largest value the defender robots can cope with before they lose their ability to accomplish a synchronous intercept. From 1000 simulations for each speed ratio, we make the minimum noise strength when a failed synchronous intercept appears as the maximum tolerable noise. Fig. 15 shows that the maximum tolerable noise for both Attackeroriented strategy and Neutral-position-oriented strate- 


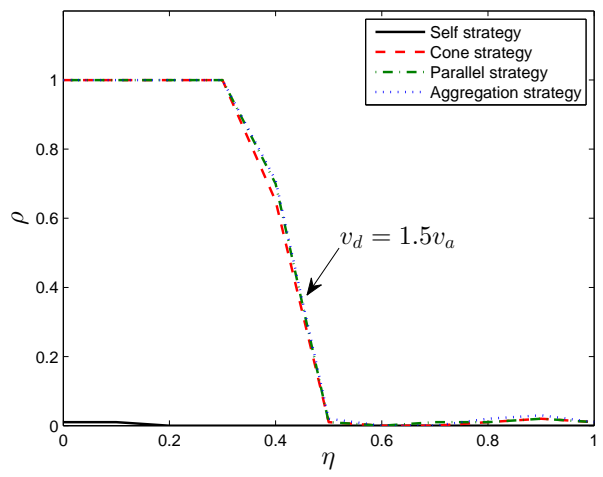

(a)

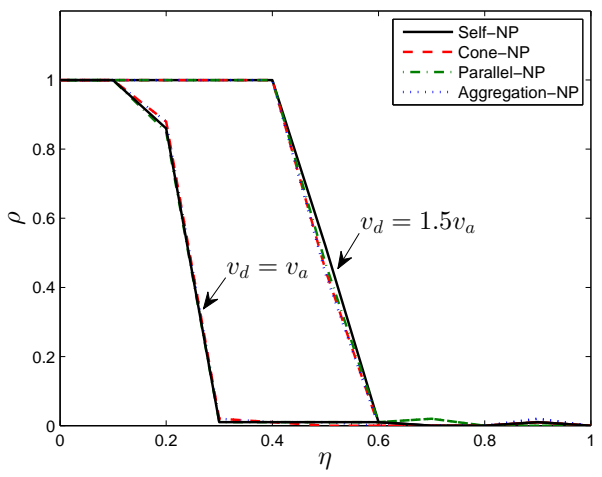

(b)

Fig. 13 The success rate $\rho$ under different noise strengthes $\eta$. (a) The success rate by using Attacker-oriented strategy under different noise strengthes. (b) The success rate by using Neutral-position-oriented strategy under different noise strengthes.

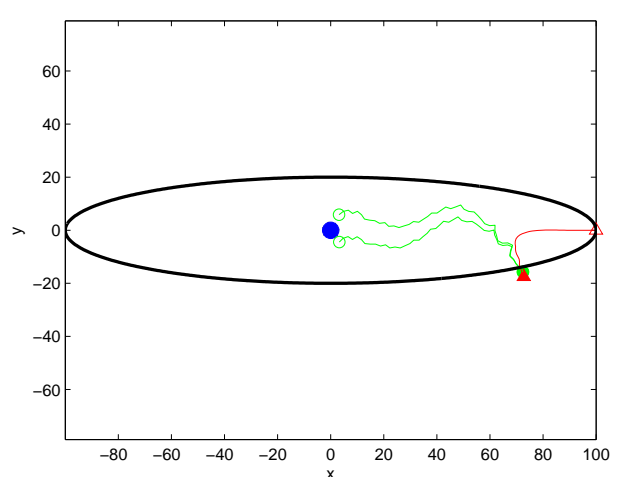

(a)

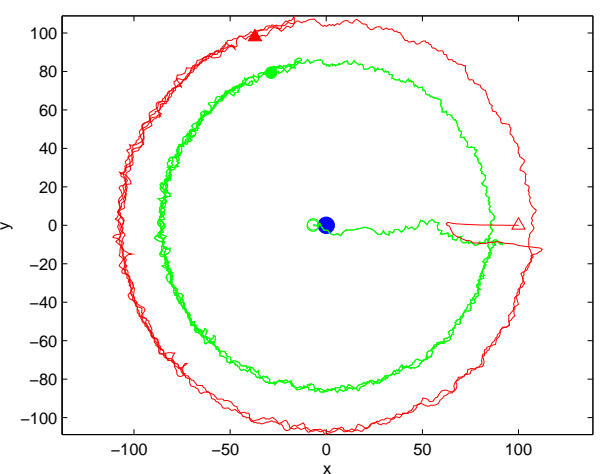

(b)

Fig. 14 Illustration of synchronous intercept patterns when noise is present. (a) A contact intercept pattern is generated by using Parallel strategy when noise is present $(\gamma=2, \eta=0.3)$. (b) A stable non-contact intercept pattern is generated by using Parallel-NP strategy when noise is present $(\gamma=2, \eta=0.4)$.

gy increases monotonically as the speed ratio increases (speed advantage over the attacker) with saturation reached at $\eta *=0.6$. Another result is that the Neutralposition-oriented strategy has a better maximum tolerable noise than that of the Attacker-oriented strategy, reaching the same saturation point at a lower speed ratio.

\section{Conclusion}

In this paper, the so-called "synchronous intercept problem" with two defender robots and one attacker robot is introduced. The attacker robot is assumed to have attraction to the target and repulsion from the defenders, while the two defender robots try to protect this target from the attacker robot. The synchronous intercept requires that both defender robots reach the attacker's location synchronously, otherwise either of

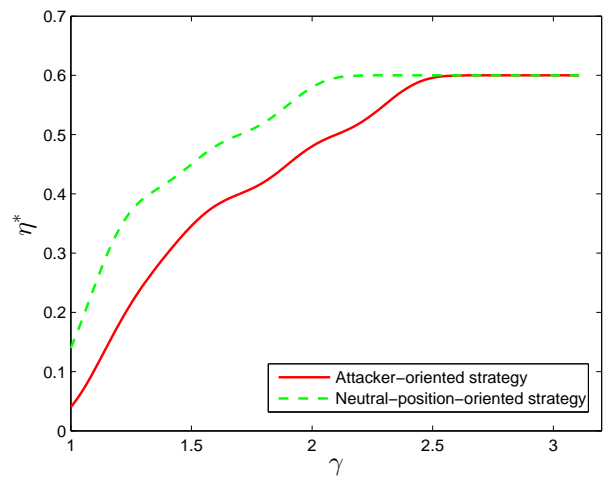

Fig. 15 Illustration of maximum tolerable noise as a function of speed ratio.

two defender robots arriving individually will get destroyed by the attacker robot. In order to accomplish this synchronous intercept mission, Attacker-oriented and Neutral-position-oriented intercept strategies are 
proposed. These strategies generate the contact intercept pattern and the stable non-contact intercept pattern, respectively. The contact intercept pattern allows the defender robots to synchronously reach the attacker robot in finite time. The stable non-contact intercept pattern is a periodic attractor, where the defender robots and the attacker robot orbit around the target with the same angular speed on two concentric circles in infinite time. Both intercept strategies need the defender robots to be deployed inside a feasible area.

Synchronous intercept is a special requirement in defense-intrusion game. This special case demands the defender robots to co-ordinate their activities. According to practical needs, contact intercept pattern and non-contact intercept pattern are generated by the proposed Attacker-oriented and Neutral-position-oriented intercept strategies, respectively. The Attacker-oriented intercept strategy is a synchronous attack-only intercept strategy, it applies to the scenario where the attacker robot has to be destroyed before it reaches the target. One of the main limits of this strategy is that, when the defender robots have the same mobility as the attacker robot, they have to be deployed symmetrically in the feasible area. The Neutral-position-oriented strategy is a more flexible intercept strategy, as it considers both the synchronous attack and defense behaviors. It has a larger feasible area and a better tolerance for noise than the Attacker-oriented strategy. Besides the scenario that the attacker robot has to be destroyed, it also applies to the scenario where preventing a suspected attacker robot from reaching the target would be a more reasonable measure than destroying them. The trade-off between attack and defense behaviors can be flexibly adjusted by the parameter $\lambda$, hence many potential intercept strategies can be designed by varying $\lambda$.

The results of the synchronous intercept strategies are provided with the case of two defenders. In ongoing and future work, we are extending the model to a general multi-defender case. The farthest defender in multi-defender case can use the control policy of the further defender in two-defender case, while the closer defenders can use the similar control policy as the closer defender in two-defender case. The scalability of the proposed algorithms is to be tested by numerical simulations and a real robotic platform with consideration of collision avoidance.

\section{Acknowledgements}

This work was partially supported by the National Natural Science Foundation of China (NSFC) under Grants 51879219, 51679201, and Science and Technology on
Underwater Information and Control Laboratory under Grants 6142218051806. We are grateful to the reviewers for their suggestions to improve this paper.

\section{References}

Alexander S, Bishop R, Ghrist R (2009) Capture pursuit games on unbounded domains. Enseign Math(2) 55(1-2):103-125

Antonelli G, Arrichiello F, Chiaverini S (2007) The entrapment/escorting mission for a multi-robot system: Theory and experiments. In: Advanced intelligent mechatronics, 2007 ieee/asme international conference on, IEEE, pp 1-6

Basar T, Olsder GJ (1999) Dynamic noncooperative game theory, vol 23. Siam

Bertram BCR (1978) Living in groups: predators and prey. In: Evolutionary Computation, 2005. The 2005 IEEE Congress on, pp $41-48$ Vol.1

Bhattacharya S, Hutchinson S (2008) Approximation schemes for two-player pursuit evasion games with visibility constraints. In: Robotics: Science and Systems

Bhattacharya S, Başar T, Hovakimyan N (2011) Singular surfaces in multi-agent connectivity maintenance games. In: Decision and Control and European Control Conference (CDC-ECC), 2011 50th IEEE Conference on, IEEE, pp 261-266

Bhattacharya S, Basar T, Hovakimyan N (2014) On the construction of barrier in a visibility based pursuit evasion game. In: Control Conference (ECC), 2014 European, IEEE, pp 1894-1901

Bopardikar SD, Suri S (2014) k-capture in multiagent pursuit evasion, or the lion and the hyenas. Theoretical Computer Science 522:13-23

Boyell RL (1976) Defending a moving target against missile or torpedo attack. IEEE Transactions on Aerospace and Electronic Systems (4):522-526

Breakwell JV (1975) Pursuit of a faster evader. In: The Theory and Application of Differential Games, Springer, pp 243-256

Chen J, Zha W, Peng Z, Gu D (2016a) Multi-player pursuit-evasion games with one superior evader. Automatica 71:24-32

Chen M, Zhou Z, Tomlin CJ (2014a) Multiplayer reachavoid games via low dimensional solutions and maximum matching. In: 2014 American control conference, IEEE, pp 1444-1449

Chen M, Zhou Z, Tomlin CJ (2014b) A path defense approach to the multiplayer reach-avoid game. In: 53rd IEEE conference on decision and control, IEEE, pp 2420-2426 
Chen M, Zhou Z, Tomlin CJ (2016b) Multiplayer reachavoid games via pairwise outcomes. IEEE Transactions on Automatic Control 62(3):1451-1457

Garcia E, Casbeer DW, Pachter M (2019) Design and analysis of state-feedback optimal strategies for the differential game of active defense. IEEE Transactions on Automatic Control 64(2):553-568

Gese EM (2001) Territorial defense by coyotes (canis latrans) in yellowstone national park, wyoming: who, how, where, when, and why. Canadian Journal of Zoology 79(6):980-987

Hagedorn P, Breakwell J (1976) A differential game with two pursuers and one evader. Journal of Optimization Theory and Applications 18(1):15-29

Huang TY, Xue-Bo C, Wang-Bao X, Zi-Wei Z, ZhiYong R (2013) A self-organizing cooperative hunting by swarm robotic systems based on loose-preference rule. Acta Automatica Sinica 39(1):57-68

Iwama T, Sato M (2012) Group chase and escape with some fast chasers. Physical Review E 86(6):067,102

Janosov M, Virágh C, Vásárhelyi G, Vicsek T (2017) Group chasing tactics: how to catch a faster prey. New Journal of Physics 19(5):053,003

Kamimura A, Ohira T (2010) Group chase and escape. New Journal of Physics 12(5):053,013

Kopparty S, Ravishankar CV (2005) A framework for pursuit evasion games in $\mathrm{rn}$. Information Processing Letters 96(3):114-122

Kothari M, Manathara JG, Postlethwaite I (2014) A cooperative pursuit-evasion game for non-holonomic systems. IFAC Proceedings Volumes 47(3):1977-1984

Li W (2016a) Escape analysis on the confinementescape problem of a defender against an evader escaping from a circular region. IEEE transactions on cybernetics 46(9):2166-2172

Li W (2016b) Formulation of a cooperativeconfinement-escape problem of multiple cooperative defenders against an evader escaping from a circular region. Communications in Nonlinear Science and Numerical Simulation 39:442-457

Liang L, Deng F, Peng Z, Li X, Zha W (2019) A differential game for cooperative target defense. Automatica 102:58-71

Littlewood JE (1986) Littlewood's miscellany. Cambridge University Press

Liu SY, Zhou Z, Tomlin C, Hedrick K (2013a) Evasion as a team against a faster pursuer. In: 2013 American control conference, IEEE, pp 5368-5373

Liu SY, Zhou Z, Tomlin C, Hedrick K (2013b) A gradient-based method for team evasion. In: ASME 2013 Dynamic Systems and Control Conference, American Society of Mechanical Engineers Digital Collection
Liu SY, Zhou Z, Tomlin C, Hedrick JK (2014) Evasion of a team of dubins vehicles from a hidden pursuer. In: 2014 IEEE International Conference on Robotics and Automation (ICRA), IEEE, pp 6771-6776

Makkapati VR, Sun W, Tsiotras P (2018) Optimal evading strategies for two-pursuer/one-evader problems. Journal of Guidance, Control, and Dynamics 41(4):851-862

Martin HJA, de Lope J, Maravall D (2010) Analysis and solution of a predator-protector-prey multirobot system by a high-level reinforcement learning architecture and the adaptive systems theory. Robotics and Autonomous Systems 58(12):12661272

Masuko M, Hiraoka T, Ito N, Shimada T (2017) The effect of laziness in group chase and escape. Journal of the Physical Society of Japan 86(8):085,002

Neill SSRJ, Cullen JM (1974) Experiments on whether schooling by their prey affects the hunting behaviour of cephalopods and fish predators. Journal of Zoology 172(4):549-569

Oyler DW, Kabamba PT, Girard AR (2016) Pursuitevasion games in the presence of obstacles. Automatica 65:1-11

Pachter M, Garcia E, Casbeer DW (2019) Toward a solution of the active target defense differential game. Dynamic Games and Applications 9(1):165-216

Pan S, Huang H, Ding J, Zhang W, Tomlin CJ, et al (2012) Pursuit, evasion and defense in the plane. In: American Control Conference (ACC), 2012, IEEE, pp 4167-4173

Peng X, Zhang S, Lei X (2016) Multi-target trapping in constrained environments using gene regulatory network-based pattern formation. International Journal of Advanced Robotic Systems 13(5):1-12

Ramana MV, Kothari M (2017) Pursuit-evasion games of high speed evader. Journal of intelligent and robotic systems 85(2):293-306

Ripple WJ, Larsen EJ (2000) Historic aspen recruitment, elk, and wolves in northern yellowstone national park, usa. Biological Conservation 95(3):361-370

Saito T, Nakamura T, Ohira T (2016) Group chase and escape model with chasers' interaction. Physica A: Statistical Mechanics and its Applications 447:172179

Shishika D, Kumar V (2018) Local-game decomposition for multiplayer perimeter-defense problem. In: 2018 IEEE Conference on Decision and Control (CDC), IEEE, pp 2093-2100

Shishika D, Paley DA (2019) Mosquito-inspired distributed swarming and pursuit for cooperative defense against fast intruders. Autonomous Robots 43(7):1781-1799 
Siegfried WR, Underhill LG (1975) Flocking as an anti-predator strategy in doves. Animal Behaviour 23(75):504-508

Sun Q, Shen M, Gu X, Hou K, Qi N (2019) Evasionpursuit strategy against defended aircraft based on differential game theory. International Journal of Aerospace Engineering 2019:7980,379

Takei R, Tsai R, Zhou Z, Landa Y (2014) An efficient algorithm for a visibility-based surveillanceevasion game. Communications in Mathematical Sciences 12(7):1303-1327

Turetsky V (2008) Capture zones of linear feedback pursuer strategies. Automatica 44(2):560-566

Vicsek T (2010) Statistical physics: Closing in on evaders. Nature 466(7302):43

Wang J, Li W (2015) Motion patterns and phasetransition of a defender-intruder problem and optimal interception strategy of the defender. Communications in Nonlinear Science and Numerical Simulation 27(1):294-301

Yang S, Jiang S, Jiang L, Li G, Han Z (2014) Aggregation increases prey survival time in group chase and escape. New Journal of Physics 16(8):083,006

Zha W, Chen J, Peng Z, Gu D (2016) Construction of barrier in a fishing game with point capture. IEEE transactions on cybernetics 47(6):1409-1422

Zhang F, Zha W (2018) Evasion strategies of a threeplayer lifeline game. Science China Information Sciences 61(11):112,206

Zhang S, Liu M, Lei X, Huang Y, Zhang F (2018) Multi-target trapping with swarm robots based on pattern formation. Robotics and Autonomous Systems 106:1-13

Zhang S, Liu M, Lei X, Huang Y (2019) Stay-eat or run-away: Two alternative escape behaviors. Physics Letters A 383(7):593-599

Zhou Z, Takei R, Huang H, Tomlin CJ (2012) A general, open-loop formulation for reach-avoid games. In: 2012 IEEE 51st IEEE Conference on Decision and Control (CDC), IEEE, pp 6501-6506

Zhou Z, Zhang W, Ding J, Huang H, Stipanović DM, Tomlin CJ (2016) Cooperative pursuit with voronoi partitions. Automatica 72:64-72

Zhou Z, Ding J, Huang H, Takei R, Tomlin C (2018) Efficient path planning algorithms in reach-avoid problems. Automatica 89:28-36 